\title{
Research Paper: \\ Developing the Persian Version of Positive and Negative Affect Scale for Children (Original and Short Form) and Assessing Its Psychometric Properties
}

\author{
Mozhgan Lotfi $^{1} \odot$, Leila Bahrampoori ${ }^{2} \odot{ }^{*}$ Mahdi Amini $^{3} \odot$, Reyhane Fatemitabar $^{2} \odot$, Behrooz Birashk $^{4} \odot$, Yasamin Shiasi $^{5}$
}

1. Department of Mental Health, School of Behavioral Sciences and Mental Health (Tehran Institute of Psychiatry), Iran University of Medical Sciences, Tehran, Iran. 2. Azad University, Science and Research Branch, Tehran, Iran.

3. Department of Addiction, School of Behavioral Sciences and Mental Health (Tehran Institute of Psychiatry), Iran University of Medical Sciences, Tehran, Iran.

4. Department of Clinical Psychology, School of Behavioral Sciences and Mental Health (Tehran Institute of Psychiatry), Iran University of Medical Sciences, Tehran, Iran.

5. Department of Clinical Psychology, University of Social Welfare and Rehabilitation Sciences, Tehran, Iran.

\begin{tabular}{c|c}
$\begin{array}{c}\text { Use vourdevice toscan } \\
\text { and read the article online }\end{array}$ \\
and Negative Affect Scale for Children (Original and Short Form) and Assessing Its Psychometric Properties .Iranian Journal of \\
Psychiatry and Clinical Psychology. 2020; 25(4):440-453. http://dx.doi.org/10.32598/ijpcp.25.4.7 \\
d cilth://dx.doi.org/10.32598/ijpcp.25.4.7
\end{tabular}

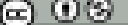

Received: 31 Oct 2018 Accepted: 13 Jul 2019 Available Online: $01 \operatorname{Jan} 2020$

Key words: Positive affect, Negative affect, PANAS-C, PANAS-C-SF, Psychometric properties

\begin{abstract}
A B S TRACT
Objectives This study aimed to develop and validate the Persian version of original and short forms of Positive and Negative Affect Schedule for Children (PANAS-C) and evaluates its psychometric properties. Methods The is a cross-sectional study conducted on male and female students of public elementary and secondary schools in Tehran, Iran in the academic year 2017-2018. The samples were 412 students (56.8\% female) selected using cluster sampling method. The data collection tools included PANAS-C, Emotion Regulation Questionnaire For Children And Adolescents (ERQ-CA), Children's Depression Inventory (CDI) and Spence Children's Anxiety Scale (SCAS). Data analysis were performed in SPSS V.20 using descriptive and inferential statistics (exploratory factor analysis, Cronbach's alpha and Pearson correlation test).

Results The factor analysis revealed that the two factors (Positive and Negative Affect) for original and short forms of PANAS-C. Cronbach's alpha for the total items of original and short forms were 0.85 and 0.81 , respectively Moreover, the correlation of positive and negative affects with reappraisal and suppression strategies, anxiety disorders (except obsessive-compulsive) and depression was significant ( $\mathrm{P}=$ 0.01, and 005).

Conclusion The Persian version of both original and short forms of PANAS-C had good psychometric properties and it can be used for research and evaluation of positive and negative affect in Iranian children and adolescents.
\end{abstract}

\section{Extended Abstract}

\section{Introduction}

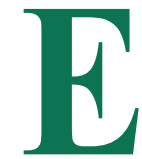

motion is a psychobiological reaction and plays an important role in human adaptation. Functionally, emotion has several positive and negative aspects. These aspects are considered as two factors/structures and supported in numer- ous studies $[1,2]$. Positive and negative affect categorized as two distinct dimensions of emotional experiences $[3,4]$. Positive affect is a state of pleasant feelings, high energy, high concentration, and pleasurable involvement. Negative affect has a state of unpleasant emotions such anger, sadness, hate, humiliation, guilt and fear $[3,5]$. The relationship of these dimensions with different psychological disorders has already been investigated [6]. There are various tools for assessing these two emotional dimensions. One

* Corresponding Author:

Mahdi Amini, PhD.

Address: Department of Addiction, School of Behavioral Sciences and Mental Health (Tehran Institute of Psychiatry), Iran University of Medical Sciences, Tehran, Iran Tel: +98 (21) 66551655-60

E-mail: amini.mh@iums.ac.ir 
of these tools is the Positive and Negative Affect Schedule for Children (PANAS-C) which is a 27-item self-report questionnaire designed by Laurent et al. for measuring the two negative and positive dimensions in children and adolescents. The psychometric properties of this scale has not been evaluated on Iranian population, and Iranian scholars use the adult version of PANAS to measure the positive and negative affect of children and adolescents. Therefore, it is necessary to use a related tool to examine children and adolescents. In this regard, the purpose of the present study was to investigate the develop and validate the Persian version of PANAS-C (original and short form) tested on Iranian school students.

\section{Methods}

The study population of this cross-sectional study consisted of all male and female students of public elementary and secondary schools in Tehran, Iran in the academic year of 2017-2018. The samples ( $\mathrm{n}=412 ; 56.8 \%$ female) were selected using cluster sampling method. For sampling, first the regions no. 2, 7 and 14 were randomly selected from 22 regions in Tehran. Then, from among the schools in each region, students of the 3 rd to 8 th grades were selected (40 per school). The data collection tools included PANASC, Emotion Regulation Questionnaire For Children And Adolescents (ERQ-CA), Children's Depression Inventory (CDI) and Spence Children's Anxiety Scale (SCAS). Data analysis were performed in SPSS v. 20 using descriptive and inferential statistics (exploratory factor analysis, Cronbach's alpha coefficient, and Pearson correlation test).

\section{Results}

The results of factor analysis showed that all factor loads were above the standard value of 0.3 . The first factor was considered positive affect and the second factor was negative affect. These two factors accounted for $51.6 \%$ of the total variance. To verify the validity of factor analysis, Cornbrash's alpha was calculated. The Cornbrash's alpha for overall PANAS-C was reported 0.85 and for both positive and negative affect factors, it was obtained 0.80 and 0.92 , respectively. The internal consistency using Cornbrash's alpha for PANAS-C-short form (PANAS-C-SF) was reported $0.81,0.79$ and 0.85 for overall, positive and negative affect, respectively. In order to determine the convergent and divergent validity of PANAS-C and PANAS-C-SF, their correlation with ERQ-CA, CDI and SCAS was examined. Results showed that PANAS-C and PANAS-C-SF factors had a significant relationship with emotion regulation, and most of depression and anxiety symptoms (except in obsessive-compulsive and fear of physical harm).

\section{Conclusion}

The PANAS-C and PANAS-C-SF had good psychometric properties to be used on Iranian children and adolescents. This indicates a favorable adaptation of this scale to Persian language and emphasizes the cross-cultural features of emotion structures.

\section{Ethical Considerations}

Compliance with ethical guidelines

The present study was designed based on the principles of the Declaration of Helsinki and was approved by Iran University of Medical Sciences.

\section{Funding}

This study was conducted with the support of Iran University of Medical Sciences.

\section{Authors contributions}

Conceptualization and investigation: Mozhgan Lotfi and Mahdi Amini; Writing: Yasamin Shiasi; Data acquisition and Analysis: Leila Bahrampoori and Reyhane Fatemitabar; Translation of the scale and interpretation: Behrooz Birashk.

\section{Conflicts of interest}

The authors declared no conflict of interest.

\section{Acknowledgements}

We want to express our gratitude towards all the study participants without whom conducting this research would not have been possible. 
This Page Intentionally Left Blank 


\title{
ويرَّى هاى روانسنجى نسخه فارسى فرم بلند و فرم كوتاه مقياس عاطفه مثبت و منفى براى كودكان
}

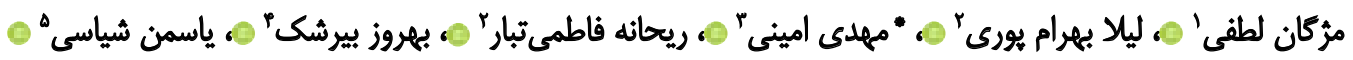 \\ ا. كروه سلامت روان، دانشكده علوم رفتارى و سلامت روان (انستيتو روانيزشكى تهران)، دانشكاه علوم يزشكى ايران، تهرانء ايران.

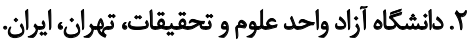

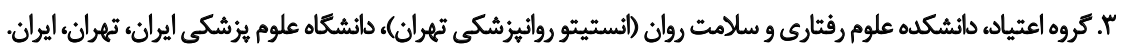

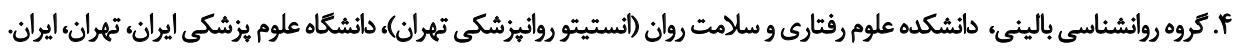

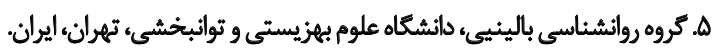

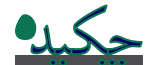

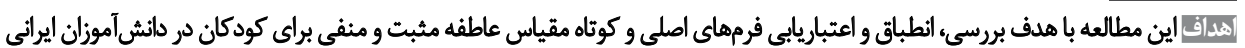

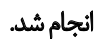

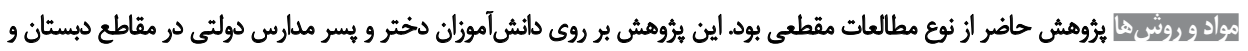

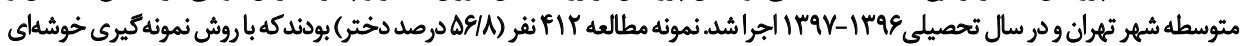

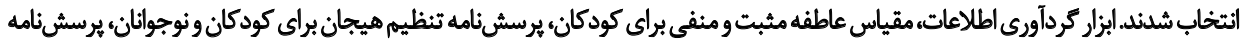

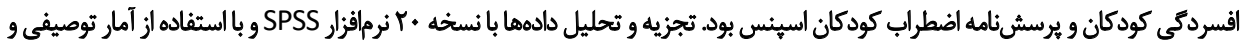

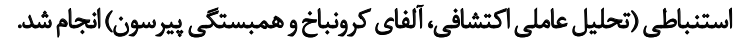

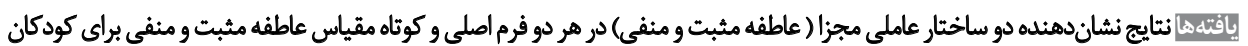

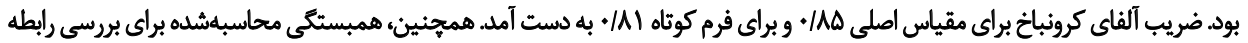

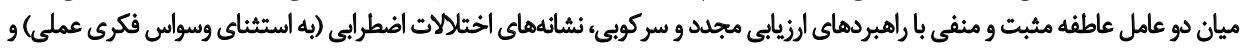

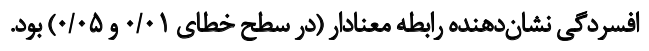

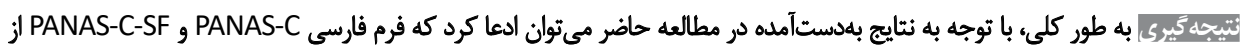

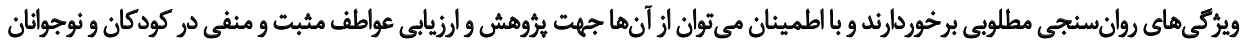
ايراثي استفاديه كرد.
\end{abstract}

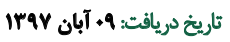

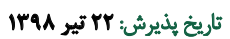

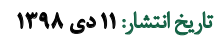

كليدوازوها:

عاطفه مثبث، عاطفه منفى،

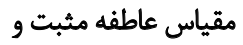

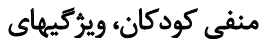
روانسينجى

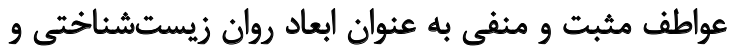

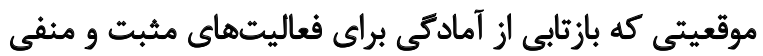

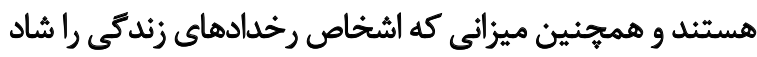

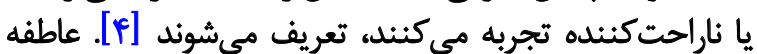

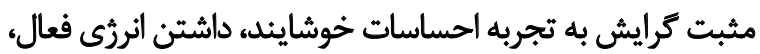

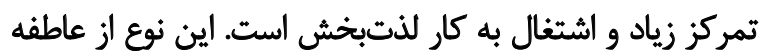

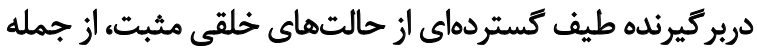

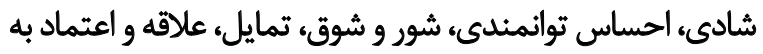

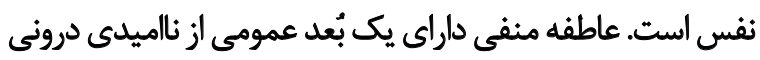

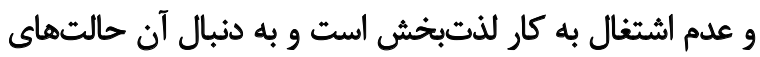

هيجان به عنوان يك واكنش زيستى روانى، كاركردهاى زيادى

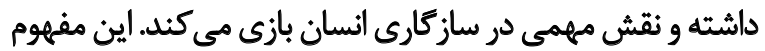

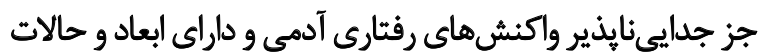

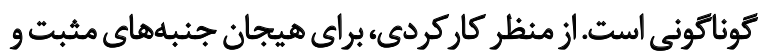

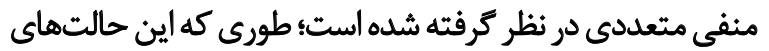

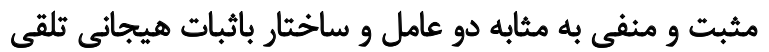

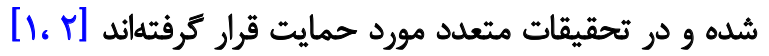

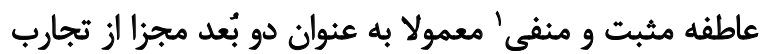

1. Positive and Negative Affect

$$
\begin{aligned}
& \text { : نويسئده مسئول: }
\end{aligned}
$$

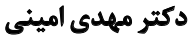

$$
\begin{aligned}
& \text { نشانى: تهران، دانشعاه علوم يزّشكى ايران، دائشكده علوم رفتّارى و سلامت روان (انستيتو روانيزشكى تهران)، كروه اعتياد. } \\
& \text { تلفن: } \\
& \text { amini.mh@iums.ac.ir بـإت الكترونيكي }
\end{aligned}
$$


معرفي شده است. مقياس عاطفه مثبت و منفى (PANAS-2) كهي

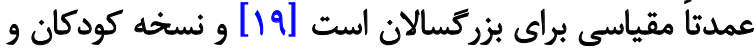

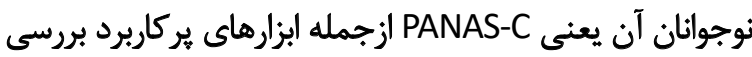

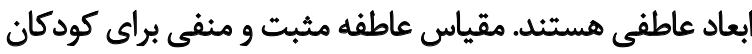

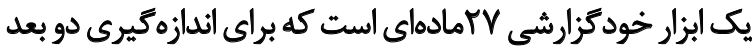

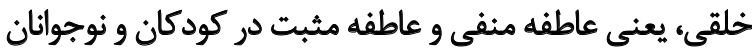

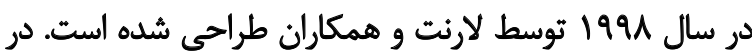

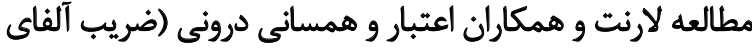

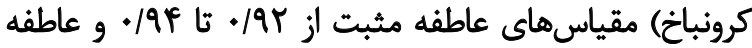

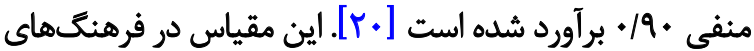

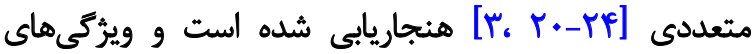

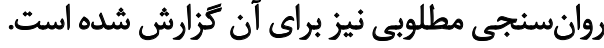

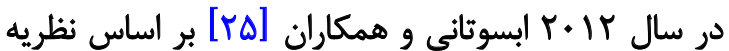

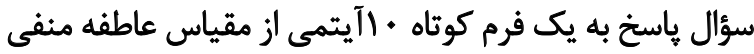

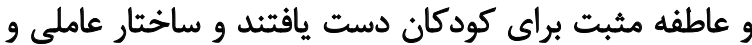

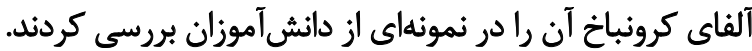

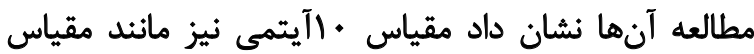

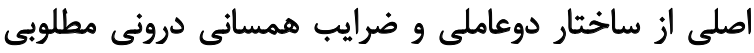

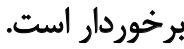

بررسى هاى انجام شده نشان مي دهد كه اين مقياس هاد ادر ايران

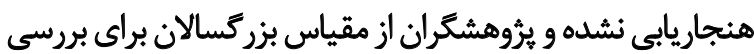

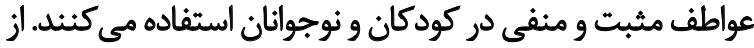

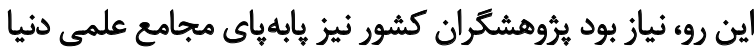

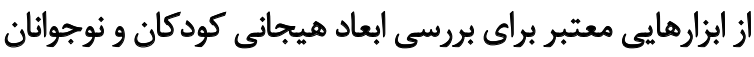

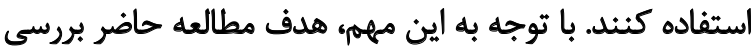

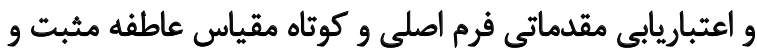

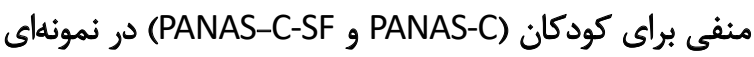

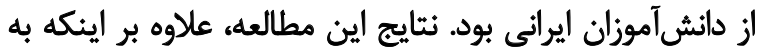

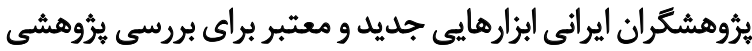

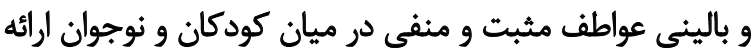

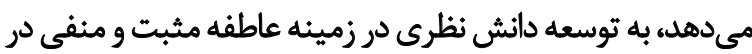
اين سنين نيز كمك شايانى مي كند.

اين مطالعه از نوع مطالعات مقطعى و با هدف انطباق

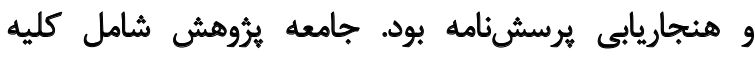

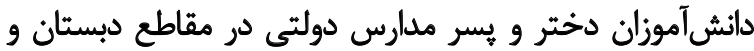

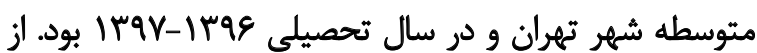

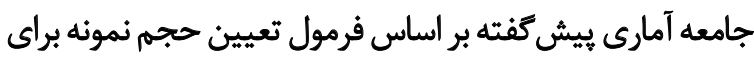

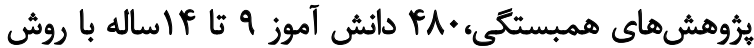

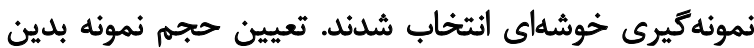

2. Positive and Negative Affect Scale
خلقى اجتنابى از قبيل حشمه، غمه، تنفر، حقارت، احساس كثناه،

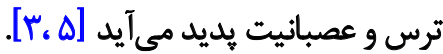

ارتباط اين دو بُعد هيجائى با بسيارى از اختلالات روانى مورد

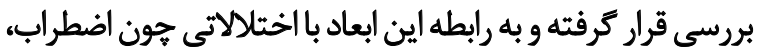

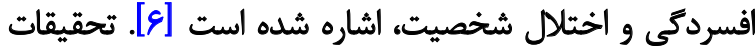

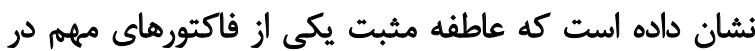

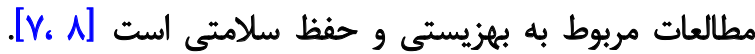
اشخاصى كه سطوح بالاترى از عواطف مثبت ورئ را تجربه مي كنيند

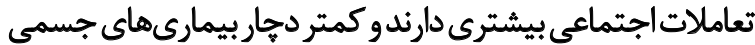

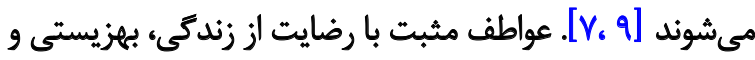

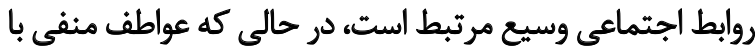

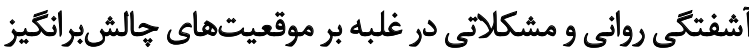

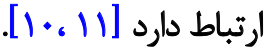

شواهد بروهشى حاكى از آن است كه بين عاطفه مثبت با باري

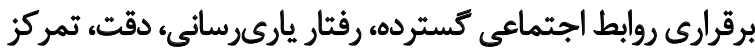

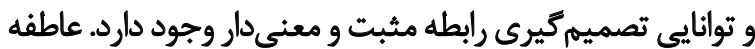

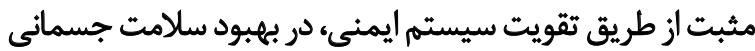

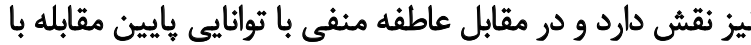

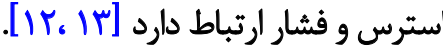

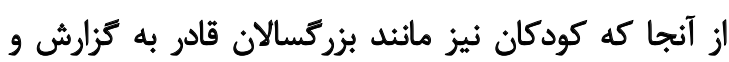

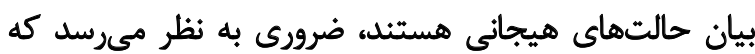

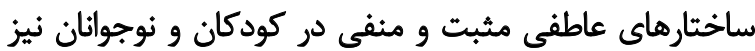

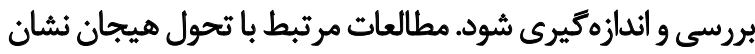

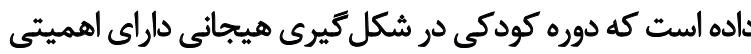

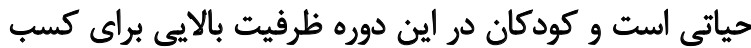

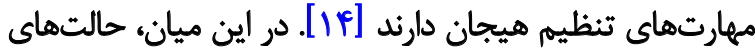

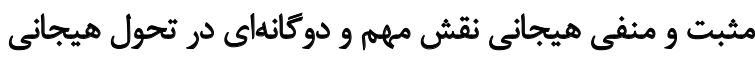

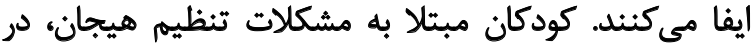

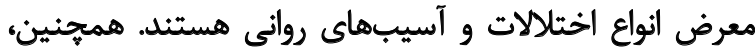

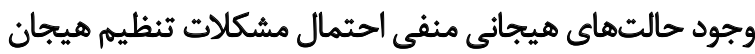

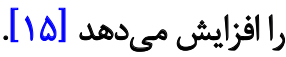
اضطراب و افسردكى از جمله اختلالات همبود رايج در إنان

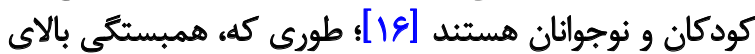

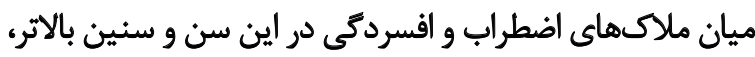

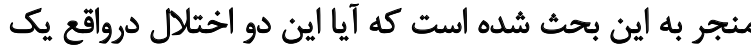

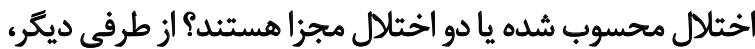

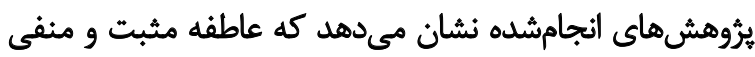

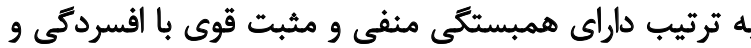

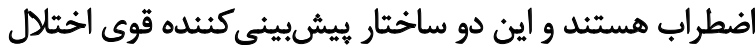

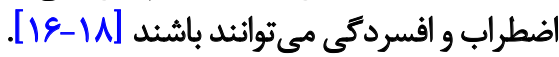
براى بررسى و مطالعه عاطفه مثبت و منفى ابزارهايى نيز 


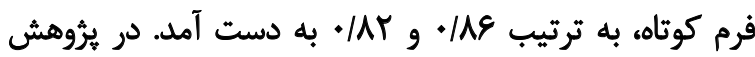

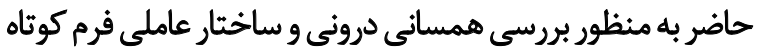

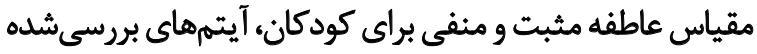

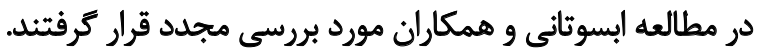

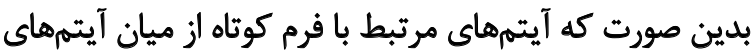

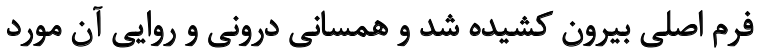

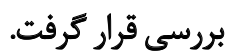

يرسشنامه تنظيم هيجان براى كودى و نوجوان (ERQ-CA):

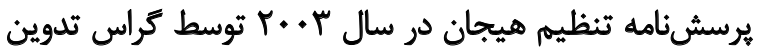

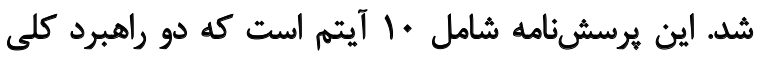

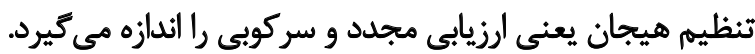

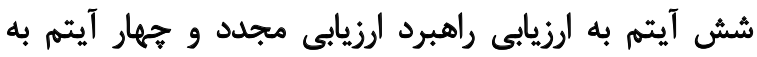

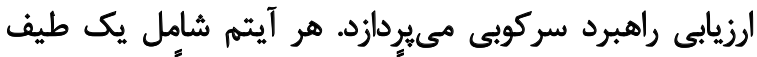

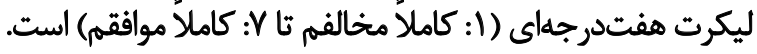

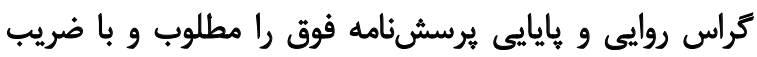

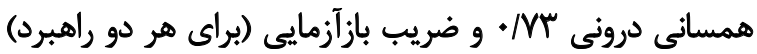

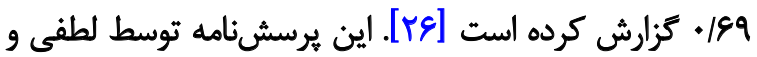

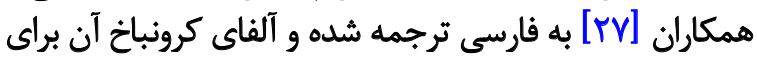

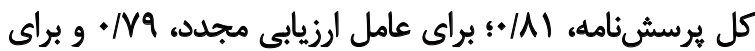

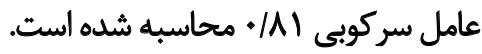

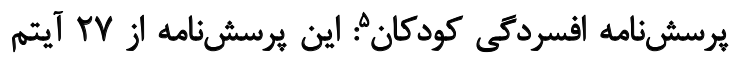

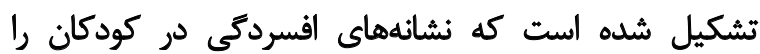

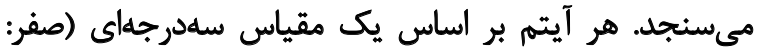

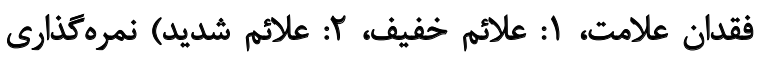

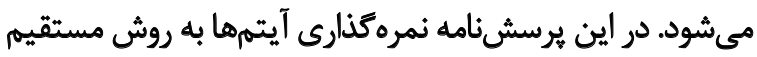

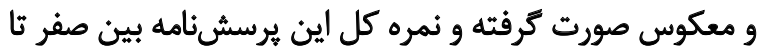

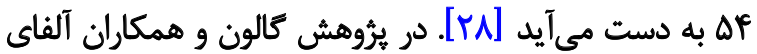

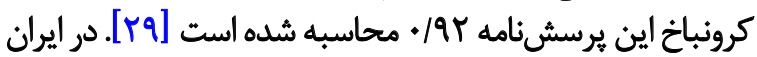

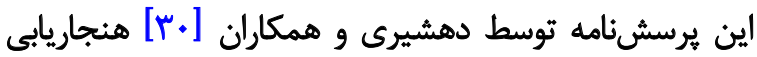

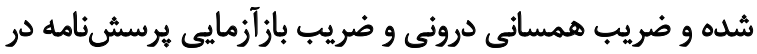

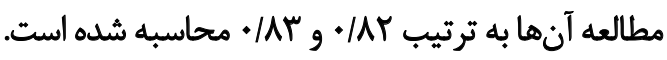

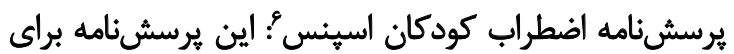

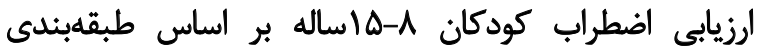

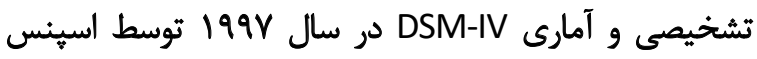

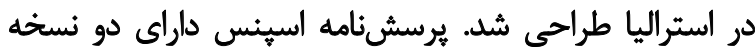

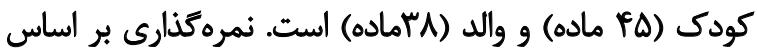

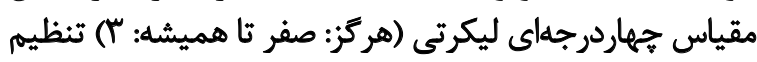

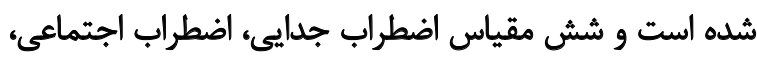

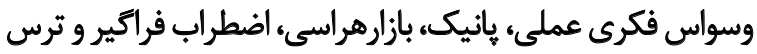

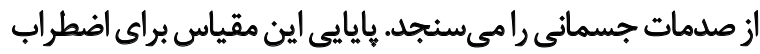

5. Children's Depression Inventory (CDI)

6. Spence Children Anxiety Scale (SCAS)

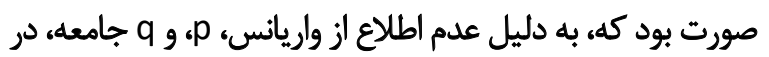

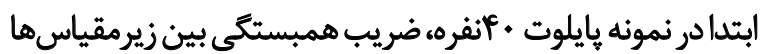

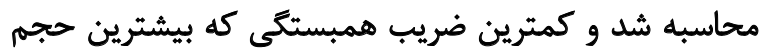

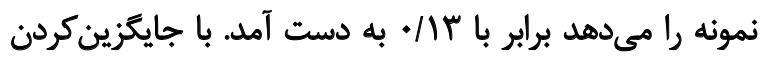

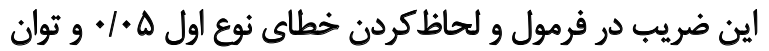

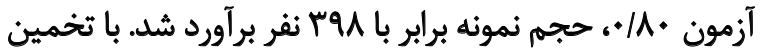

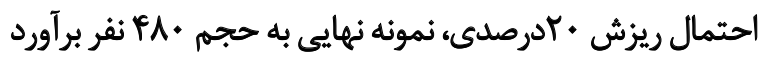

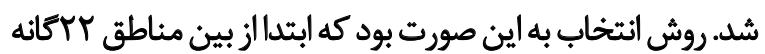

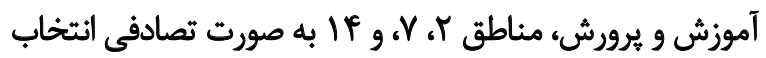

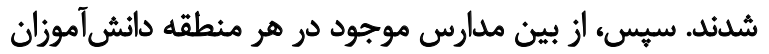

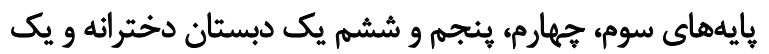

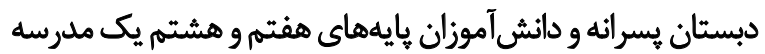

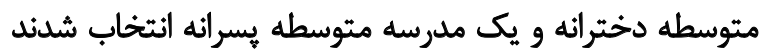

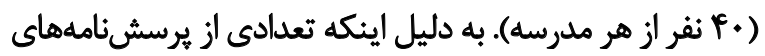

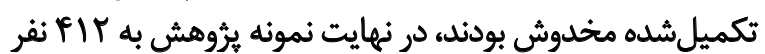

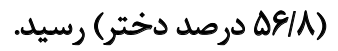

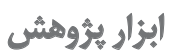

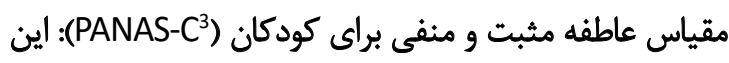

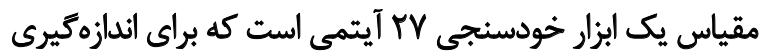

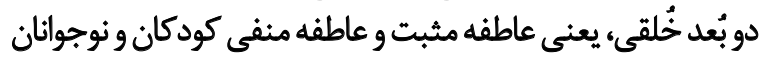

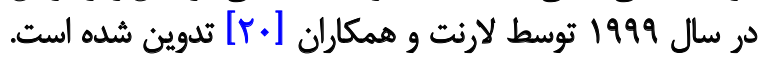
درواقع آيتمهاى PANAS-C بر اساس مقياس

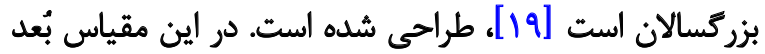

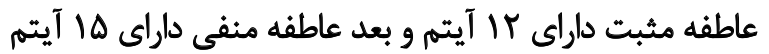

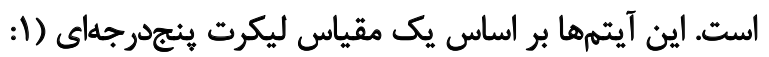

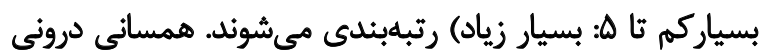

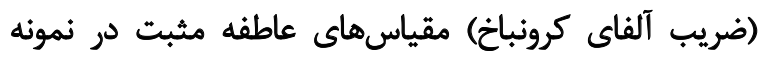

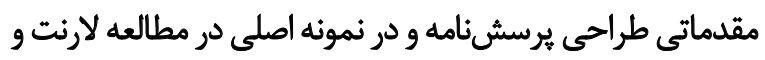

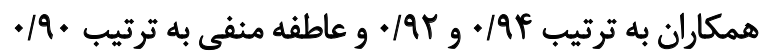

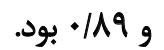

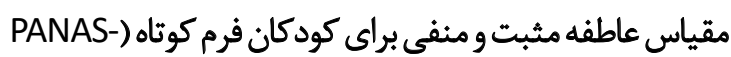
CC-SF

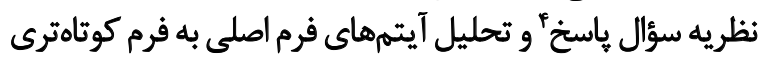

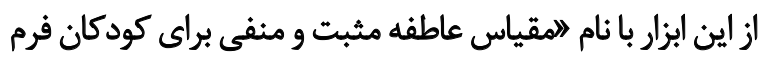

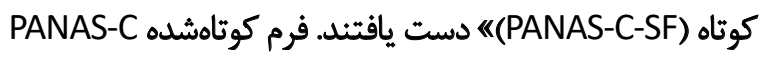
تنها • آيتم دارد (ينج آيتم براي عاطفه مثبت و وينج آيتم براي

\section{عاطفه منفى).}

در مطالعه ابسوتانى و همكاران ضريب همسانى درونى با ماني

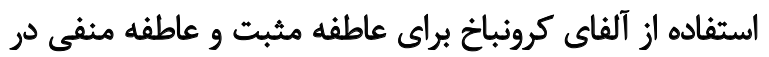

3. Positive and Negative Affect Scale

4. Item response theory 


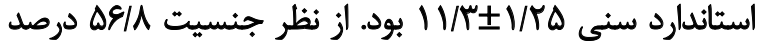

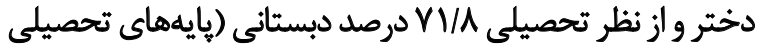

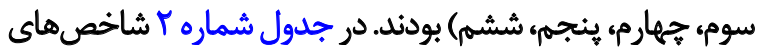

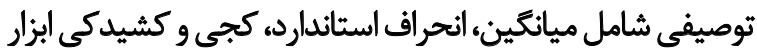
ئروهش و عوامل آنها ارائه شده است.

جهت تعيين ساختار عاملى مقياس PANAS-C، از تحليل

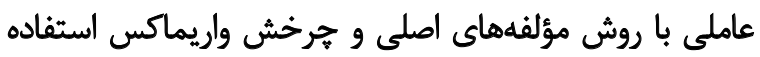

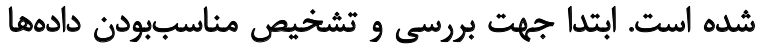

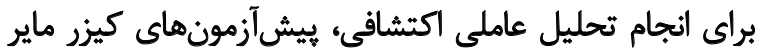

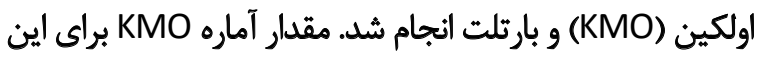

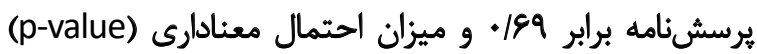

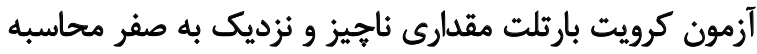

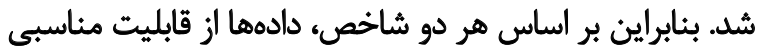

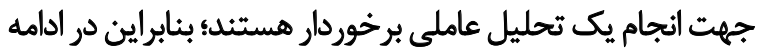

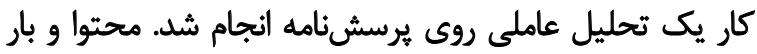

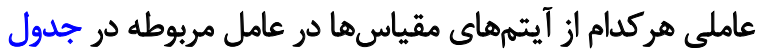

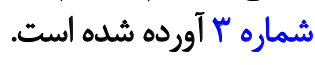

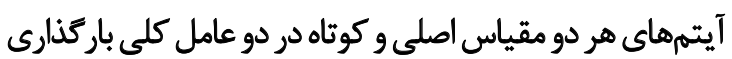

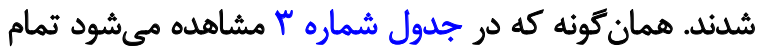

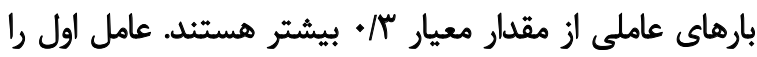

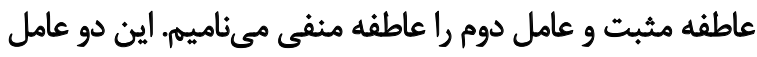

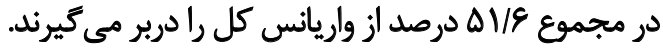
براى بررسى اعتبار تحليل عاملى، ضريب آلفاى كرونباخ براى

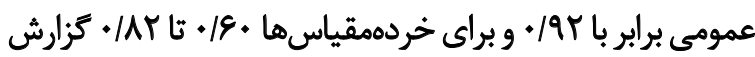

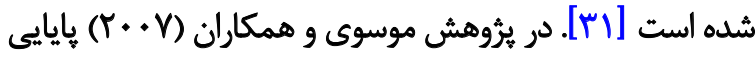

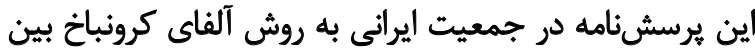

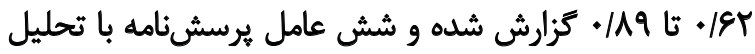

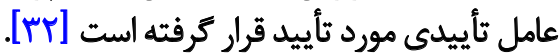

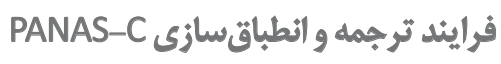

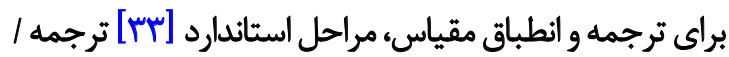

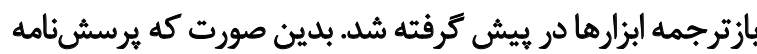

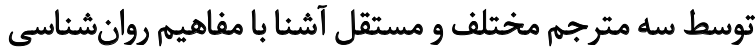

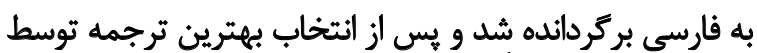

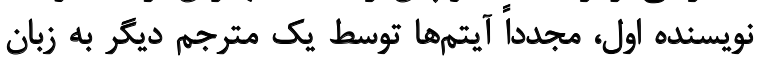

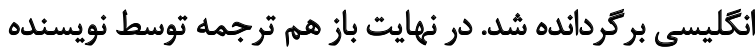

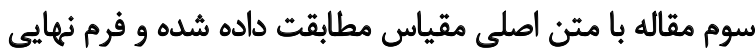

$$
\text { تلدوين شد. }
$$

براى تجزيه و تحليل دادهها از آمار توصيفى و آمار استنباطى فئى

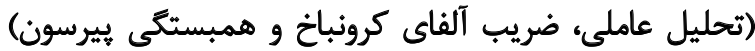

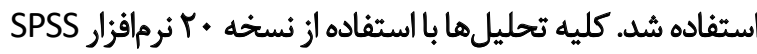

$$
\text { انجام شدند. }
$$

ياقتهها

در جدول شماره | به وضعيت جمعيتشناختى نمونه مورد

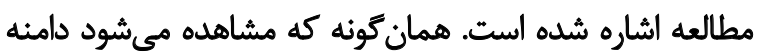

\begin{tabular}{|c|c|c|}
\hline فراوانى (درصد) & & \\
\hline$\Lambda \cdot(12 / 4)$ & 9 & \multirow{7}{*}{ سن } \\
\hline$V \Delta(N T)$ & 1. & \\
\hline$V \cdot(I V)$ & 11 & \\
\hline$V(I V / T)$ & ir & \\
\hline$n(I V / M)$ & ir & \\
\hline$\mu \omega(1 . / \Delta)$ & if & \\
\hline $\operatorname{Pir}(1 .)$. & كل & \\
\hline$m(\Delta / /)$ & لهتر & \multirow{3}{*}{ جنسيت } \\
\hline $\operatorname{IVA}(P T / N)$ & يسر & \\
\hline $\operatorname{Pir}(1 .)$. & كل & \\
\hline$r \cdot v(r / A)$ & دبستان & \multirow{3}{*}{ تحصيلات } \\
\hline $\operatorname{IV}(T N T)$ & مثوسطه & \\
\hline $\operatorname{Pir}(1 .)$. & كل & \\
\hline
\end{tabular}

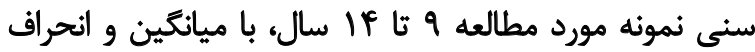

جدول ا. وضعيت جمعيتشئاختى نمونه يُروهش 
جدول r. شاخص هاى توصيفى ابزارهاى يُروهش

\begin{tabular}{|c|c|c|c|}
\hline كشيدكى & $\begin{array}{c}\text { كجى } \\
\text { (Skew) }\end{array}$ & ميانكين Iانحراف اسثاندارد & يرسش بامه/عامل \\
\hline$-\cdot / M V$ & $\Delta V$ & ST/ATIIV/AF & PANAS-C \\
\hline$\cdot / 1$ & $\cdot / M$ & $F \cdot(\Delta) \pm \cdot / 8$ & عاطفه مثبت \\
\hline$-\cdot / M^{c}$ & $+/ \Delta \Delta$ & MT/KEIV\&E & عاطفه مثقى \\
\hline$\cdot|A|$ & .11 & TV/TYIE/VE & ERQ-CA \\
\hline.$/ \Delta A$ &.$/ 2 V$ & $\mid f / \Delta f \pm f / M q$ & ارزيابي هجلد \\
\hline.$/ 79$ &.$/ M$ & $I r / M E \pm r / M$ & 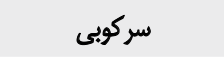 \\
\hline.$/ F r$ & - Ige & RT/VADYI/PT & SCAS \\
\hline - lar & $\cdot / \pi V$ & $V / M F \pm P / P T$ & اضطراب جدايي \\
\hline $1 / 19$ & $+/ 1$ & $N \cdot \Delta \pm \varphi / m V$ & وسواس فكرى عملى \\
\hline-+100 & $\cdot / M$ & $V / P f \pm P / \Delta f$ & ترس اجتماعى \\
\hline $1 / F V$ & $1 / 19$ & S/AY $\pm F / T \Delta$ & ترس از فضاى باز \\
\hline.$- / 40$ &.$/ r \Lambda$ & E/VE $\pm r / q r$ & اضطراب عمومى \\
\hline.$/ 10$ & $+/ \Delta A$ & $\Delta / \& \& \pm r / N$ & ترس از أسيب فيزيكى \\
\hline $1 / 8$ &.$- / . r$ & SV/RTITI/GV & CDI \\
\hline
\end{tabular}

CDI: Children's Depression Inventory SCAS:Spence Children Anxiety Scale

ERQ-CA: Emotion Regulation Questionnaire- Children-Adolescence

\section{SCAS:Spence Children Anxiety Scale}

CDI:The Children's Depression Inventory

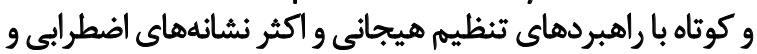

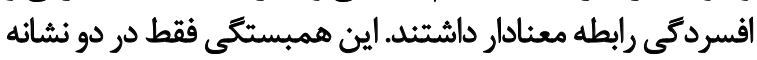

وسواس فكرى عملى و ترس از آسيب فيزيكى معنادار نبود.

$\leftrightarrow$

مطالعه حاضر با هدف انطباق، اعتباريابى و بررسى مقايسهاى فرمهاى اصلى و كوتاه مقياس عاطفه مثبت و منفى براي كود كودان

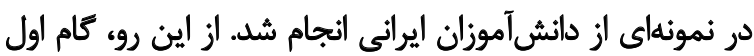

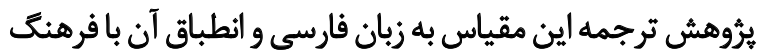

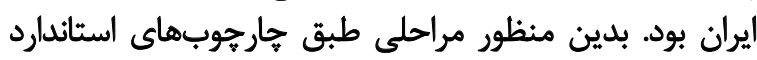

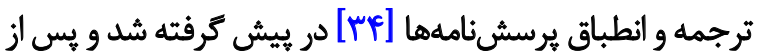

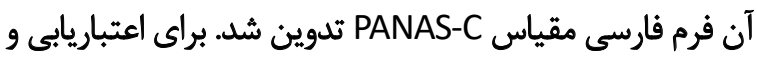

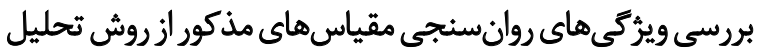
عاملي و تعيين ضريب همساني دروني با روش هي آلفاي كرونبان

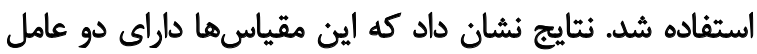

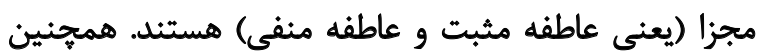

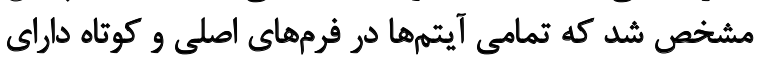

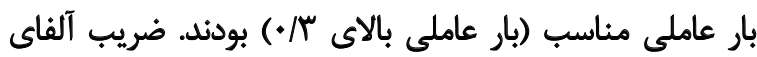

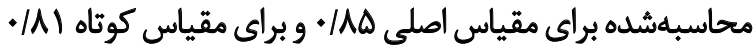
بود. اين ضرايب نشاندهنده همسانى درونى مطلوب براي نسخي إنه
كل آيتهمهاي دو مقياس و همجنين عوامل دوكانه آنها به طور

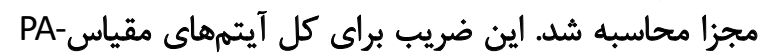

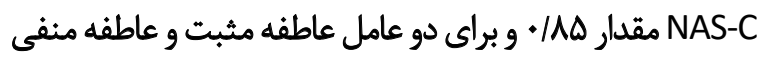

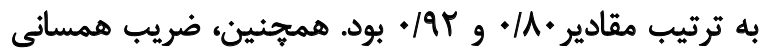

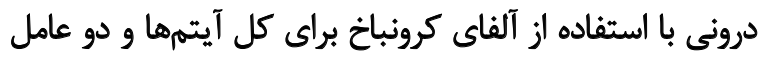

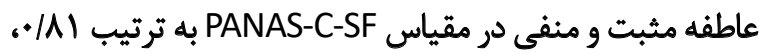

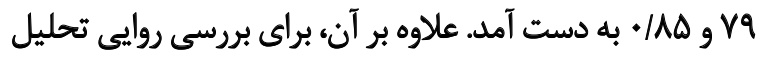

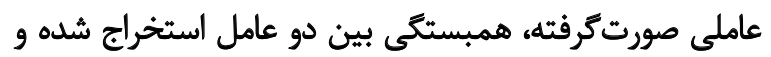

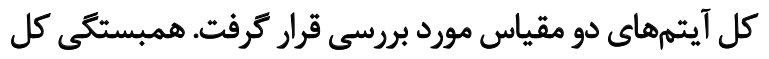

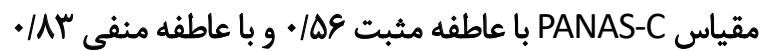

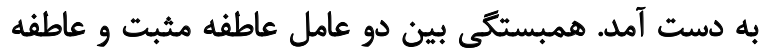

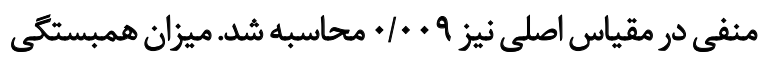

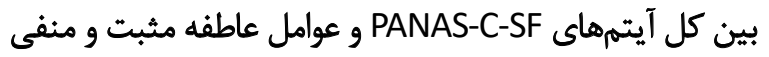

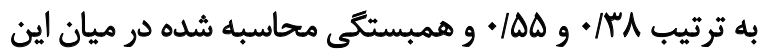

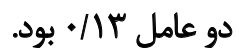

به منظور تعيين روايي همكرا و واكراي دو فرم اصلي و كوتاه

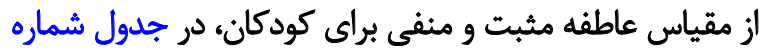

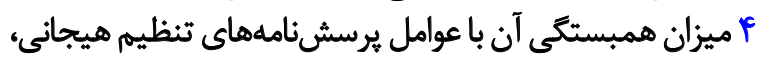

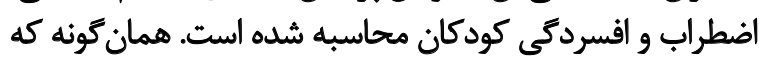

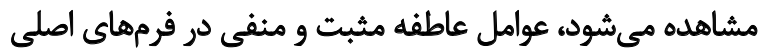


جدول ץ. ميزان بار عاملى آيتمها در مقياس اصلى و كوتاه

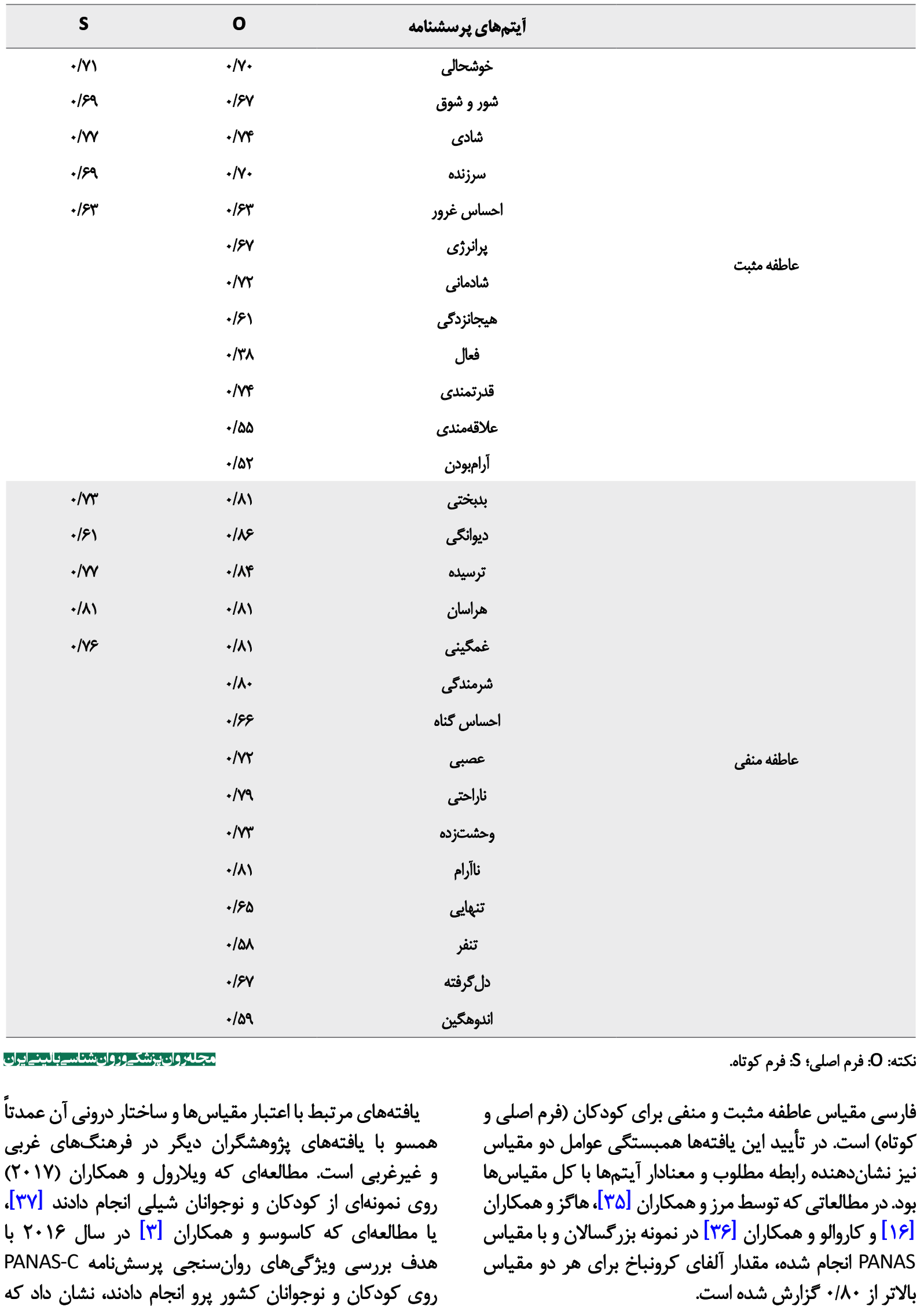


جدول F. همبستكى عاطفه مثبت و مثفى در فرم اصلى و فرم كوتاه مقياس با راهبردهاى تنظيم هيجانى، اضطراب و افسردكى

\begin{tabular}{|c|c|c|c|c|}
\hline \multicolumn{2}{|c|}{ عاطفه منفى } & \multicolumn{2}{|c|}{ عاطفه مثبت } & \multirow{2}{*}{ راهبردها / نشانعُان } \\
\hline $\mathbf{S}$ & 0 & $s$ & 0 & \\
\hline$-* / 1 \% q^{*}$ & $-\star / \& \Lambda^{*}$ &.$/ 4 r^{*}$ & $\cdot 1 \Delta F^{\circ}$ & ارزيابى مجلد \\
\hline.$/ r r$ & $+/\left.4\right|^{*}$ & $-\cdot / \Delta F^{*}$ & $-+/ \phi^{*}$ & سركوبى \\
\hline.$/ i^{*}$ &.$/ m e$ &.$- / \mathrm{A}^{* *}$ & $=* / \pi \Delta^{*}$ & اضطراب جدايع \\
\hline $.1 \cdot v$ &.$/ 1 F$ & -1.9 &.$- / 9 \cdot *$ & وسواس فكرى عملى \\
\hline.$/ 48 *$ & $\cdot / \mathrm{H}^{*}$ &.$- / 19 * *$ & $-* / M r^{* * *}$ & ترس اجتماعى \\
\hline.$/ T \Delta^{*}$ &.$/ \pi{ }^{\prime}$ &.$- / 1 V^{m e n}$ & $-\bullet / 10^{* *}$ & هراس و ترس از فضاى باز \\
\hline.$/ \Delta f^{*}$ &.$/\left.9\right|^{*}$ & $-\cdot / \Delta V^{*}$ & $--/ \Delta \Delta^{* *}$ & اضطراب فراكير \\
\hline.$/ M$ & $\cdot M I$ & $-* / 11$ & $-+/ 18$ & ترس از آسيب فيزيكى \\
\hline.$/ 48$ &.$/ \Delta e^{*}$ & $-*(+)^{*}$ & $-* / 8 q *$ & أفسردكى \\
\hline
\end{tabular}

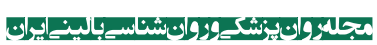

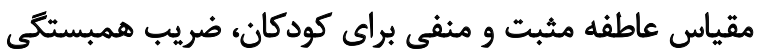

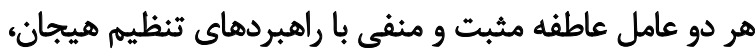

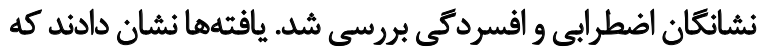

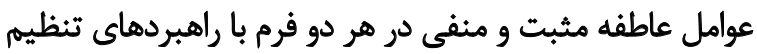

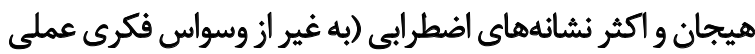

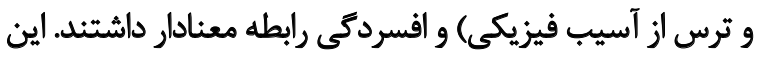

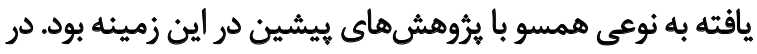

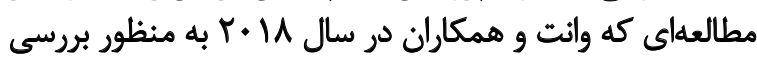

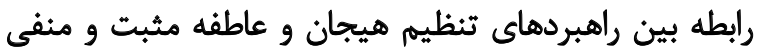

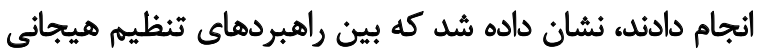

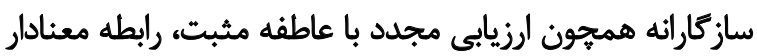

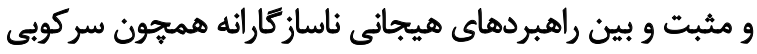

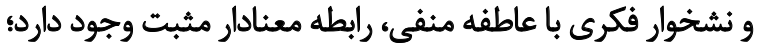

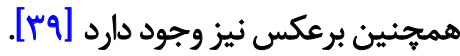

در اين مطالعه بين عوامل عاطفه مثبت و منفى با وسواس

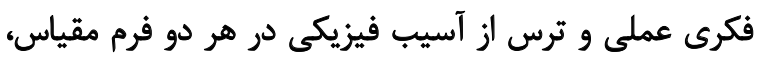

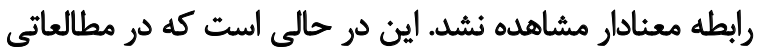

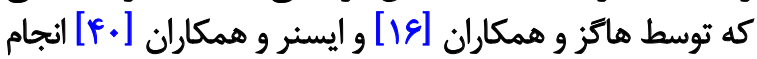

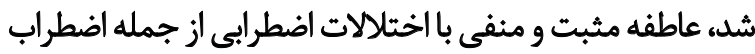

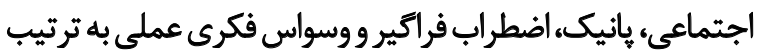

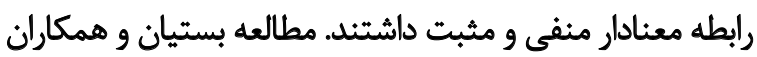

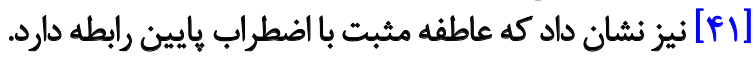

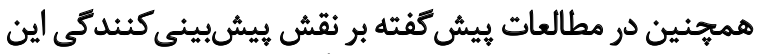

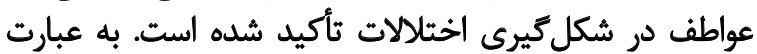

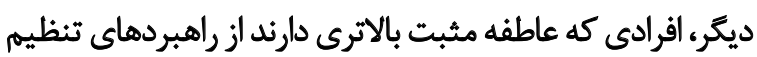

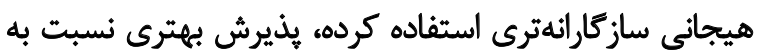

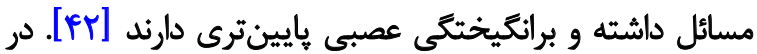

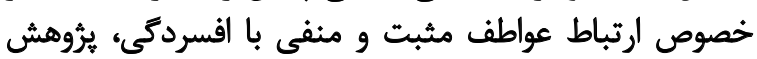

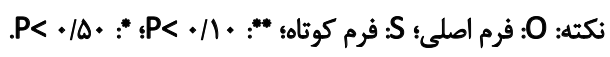

مقياس عاطفه مثبت و منفى براى كودكان در اين فرهنكَها نيز

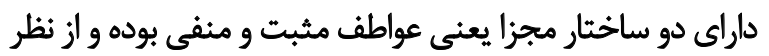

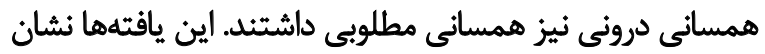

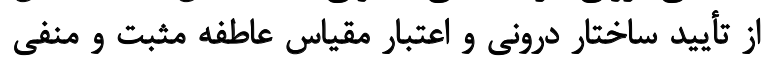

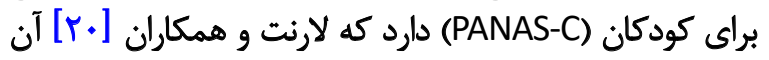

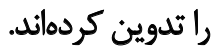

نكته قابل توجه در اين مطالعه، بررسى و مقايسه ساختار، اعتبار

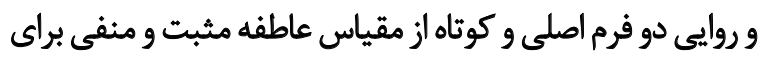

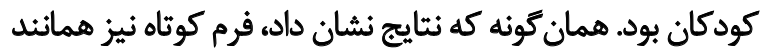

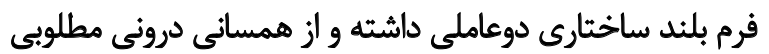

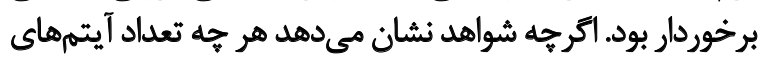

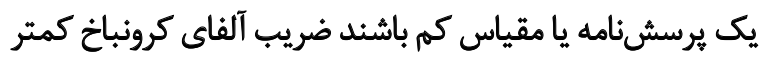

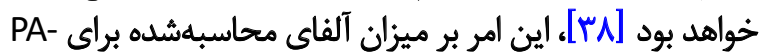
NAS-C-SF

در خصوص همسانى درونى و ساختار عاملى فرم كوثاه اين

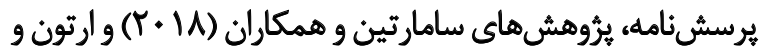

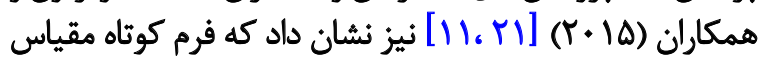

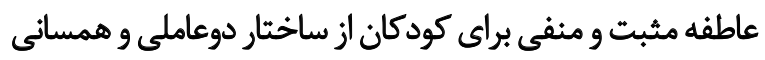

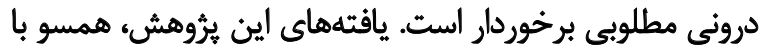

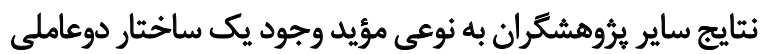

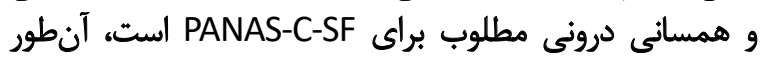

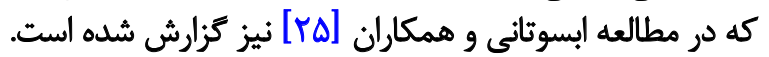

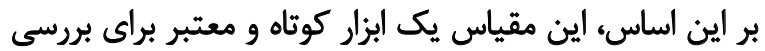

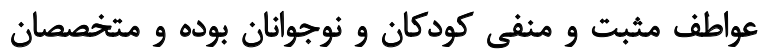

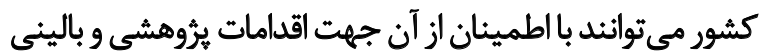
استفاده كنيند.

براى بررسى روايى هم كرا و واكراى دو فرم اصلى و كوتاه از 
سال، اشاره كرد. ييشنهاد مي شود در تحقيقات آتى يثروهشكران

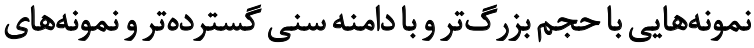

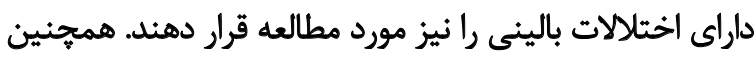

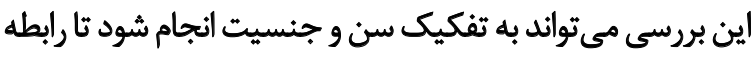

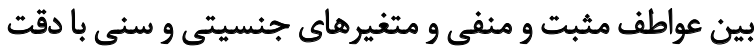
مورد مطالعه قرار كيرد.

ماحظات اخلاقى - احي

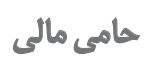

ايـن مقالـه بـا مشـاركت و حمايست دانشـعاه علـوم يزشكـى

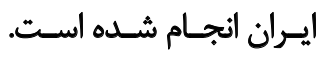

$$
\text { مشاركت ثويسند مان }
$$

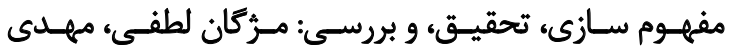

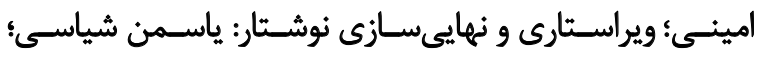

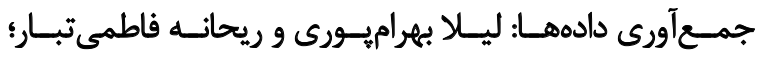

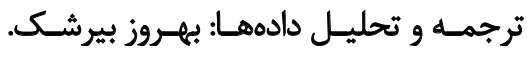

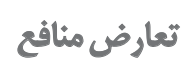

نويسندكان اذعان مي دارند در خصوص اين يُروهش هيجكونه

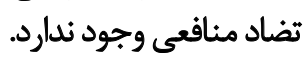

$$
\text { تشكر وقبدرافي }
$$

نويسندكان بر خود لازم مى دانيد از كليه كسانى كه در اين يُؤش يارى رسان ما بودند تشكر و قدردانى كنيند.

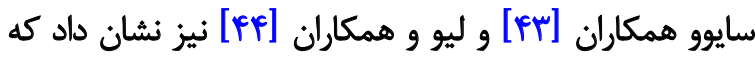

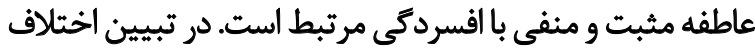

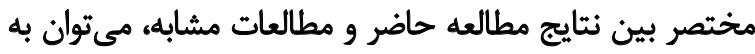

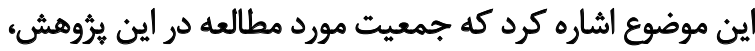

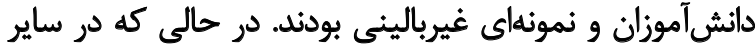

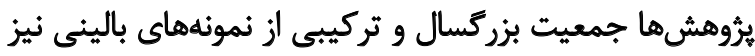

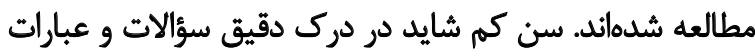

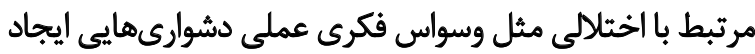
كند كه در اين زمينه نيازمئ اطلاعات بيشت فيشترى هستيه.

فمان كونه كه بيشتر كفته شده نتايج مطالعه حاضر نشان داد

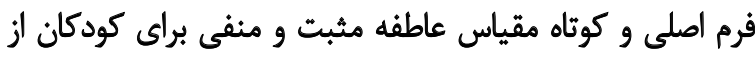

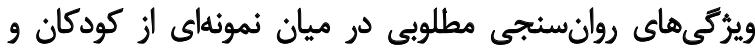

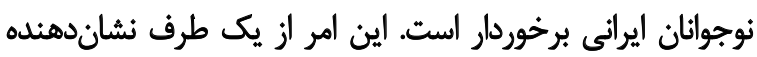

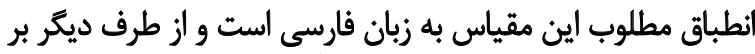

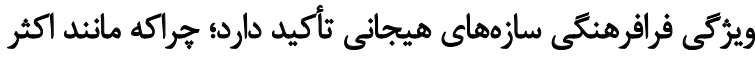

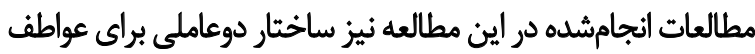

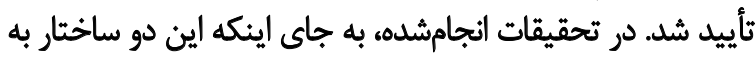

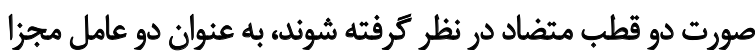

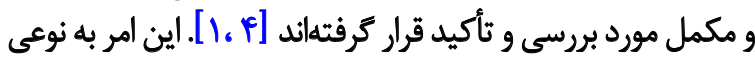

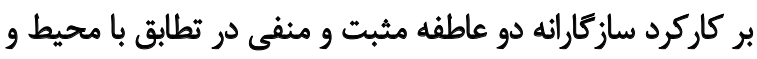

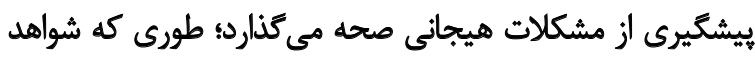

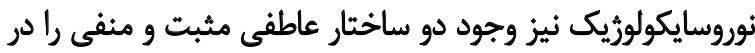

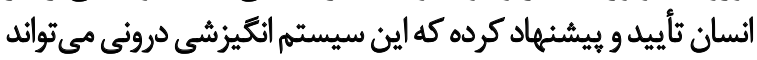

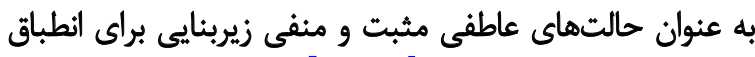

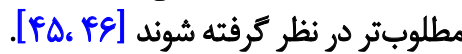

$$
\text { ثنيجليرى }
$$

به طور كلى نتايج مطالعه حاضر نشان داد كه نسخه فارسى PANAS-C-SF , PANAS-C

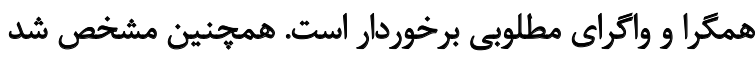

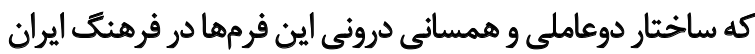

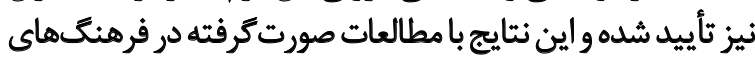

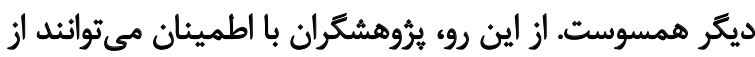

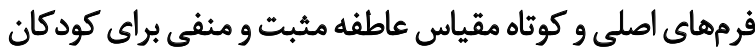

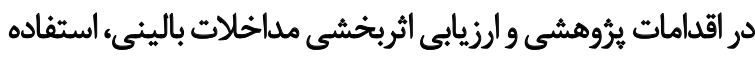
كنيند

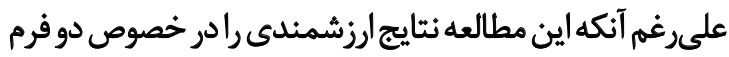

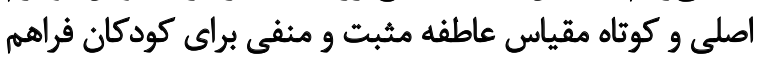

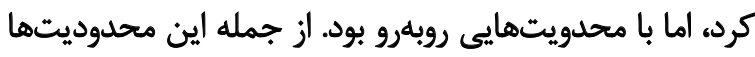

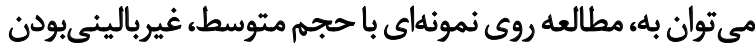

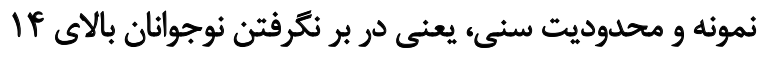

7. Positive and Negative Affect Scale-Short Form 


\section{References}

[1] Watson D, Clark LA. The PANAS-X: Manual for the positive and negative affect schedule - expanded form. Iowa Research Online; 1994. http://ir.uiowa.edu/psychology pubs/11

[2] Mackinnon A, Jorm AF, Christensen H, Korten AE, Jacomb PA, Rodgers B. A short form of the positive and negative affect schedule: Evaluation of factorial validity and invariance across demographic variables in a community sample. Personality and Individual Differences. 1999; 27(3):405-16. [DOI:10.1016/S01918869(98)00251-7]

[3] Casuso L, Gargurevich R, Van den Noortgate W, Van den Bergh O. Psychometric properties of the Positive and Negative Affect Scale for Children (PANAS-C) in Peru. Revista Interamericana de Psicologia/Interamerican Journal of Psychology. 2016; 50(2):17085.

[4] Watson D, Wiese D, Vaidya J, Tellegen A. The two general activation systems of affect: Structural findings, evolutionary considerations, and psychobiological evidence. Journal of Personality and Social Psychology. Journal of Personality and Social Psychology. 1999; 76(5):820-38. [DOI:10.1037/0022-3514.76.5.820]

[5] Quiceno JM, Vinaccia S. [Quality of life in adolescents: Analysis from personal strengths and negative emotions (Spanish)] Terapia Psicológica. 2014; 32(3):185-200. [DOI:10.4067/S071848082014000300002]

[6] Leue A, Lange S. Reliability generalization: An examination of the positive affect and negative affect schedule. Assessment. 2010; 18(4):487-501. [DOI:10.1177/1073191110374917] [PMID]

[7] Diener E, Oishi S, Lucas RE. Personality, culture, and subjective well-being: Emotional and cognitive evaluations of life. Annual Review of Psychology. 2003; 54:403-25. [DOI:10.1146/annurev. psych.54.101601.145056] [PMID]

[8] Wolniewicz CA, Tiamiyu MF, Weeks JW, Elhai JD. Problematic smartphone use and relations with negative affect, fear of missing out, and fear of negative and positive evaluation. Psychiatry Research. 2018; 262:618-23. [DOI:10.1016/j.psychres.2017.09.058] [PMID]

[9] Pettit JW, Kline JP, Gencoz T, Gencoz F, Joiner Jr TE. Are happy people healthier? The specific role of positive affect in predicting self-reported health symptoms. 2001; 35(4):521-36. [DOI:10.1006/ jrpe.2001.2327]

[10] Chavarría MP, Barra E. [Life satisfaction in adolescents: Relationship with self-efficacy and perceived social support (Spanish)]. Terapia Psicológica. 2014; 32(1):41-6. [DOI:10.4067/S071848082014000100004]

[11] Sanmartin R, Ingles CJ, Vicent M, Gonzalvez C, Diaz-Herrero A, Garcia-Fernandez JM. Positive and negative affect as predictors of social functioning in Spanish children. PloS One. 2018; 13(8):e0201698. [DOI:10.1371/journal.pone.0201698] [PMID] [PMCID]

[12] Spindler H, Denollet J, Kruse C, Pedersen SS. Positive affect and negative affect correlate differently with distress and health-related quality of life in patients with cardiac conditions: Validation of the Danish Global Mood Scale. Journal of Psychosomatic Research. 2009; 67(1):57-65. [DOI:10.1016/j.jpsychores.2008.11.003] [PMID]
[13] Hamilton JL, Burke TA, Stange JP, Kleiman EM, Rubenstein LM, Scopelliti KA, et al. Trait affect, emotion regulation, and the generation of negative and positive interpersonal events. Behavior Therapy. 2017; 48(4):435-47. [DOI:10.1016/j.beth.2017.01.006] [PMID] [PMCID]

[14] Eisenberg N, Spinrad TL, Eggum ND. Emotion-related selfregulation and its relation to children's maladjustment. Annual Review of Clinical Psychology. 2010; 6:495-525. [DOI:10.1146/annurev.clinpsy.121208.131208] [PMID] [PMCID]

[15] Cole PM, Dennis TA, Smith-Simon KE, Cohen LH. Preschoolers' emotion regulation strategy understanding: Relations with emotion socialization and child self-regulation. Social Development. 2009; 18(2):324-52. [DOI:10.1111/j.1467-9507.2008.00503.x]

[16] Hughes AA, Kendall PC. Psychometric properties of the Positive and Negative Affect Scale for Children (PANAS-C) in children with anxiety disorders. Child Psychiatry and Human Development. 2009; 40(3):343-52. [DOI:10.1007/s10578-009-0130-4] [PMID]

[17] Crawford JR, Henry JD. The Positive and Negative Affect Schedule (PANAS): Construct validity, measurement properties and normative data in a largenon-clinical sample. British Journal of Clinical Psychology. 2004; 43(3):245-65. [DOI:10.1348/0144665031752934] [PMID]

[18] Ahmadian Vargahan F, Gharraee B, Atef Vahid MK, Habibi $\mathrm{M}$. [The role of perfectionism dimensions and emotion regulation strategies in predicting the severity of depressive and anxiety symptoms of university students (Persian)]. Iranian Journal of Psychiatry \& Clinical Psychology. 2014; 20(2):153-61

[19] Watson D, Clark LA, Tellegen A. Development and validation of brief measures of positive and negative affect: The PANAS scales. Journal of Personality and Social Psychology. 1988; 54(6):1063-70. [DOI:10.1037/0022-3514.54.6.1063] [PMID]

[20] Laurent J, Catanzaro SJ, Joiner Jr TE, Rudolph KD, Potter KI Lambert $S$, et al. A measure of positive and negative affect for children: Scale development and preliminary validation. Psychological Assessment. 1999; 11(3):326-38. [DOI:10.1037/10403590.11.3.326]

[21] Ortuno-Sierra J, Santaren-Rosell M, Albeniz AP, FonsecaPedrero E. Dimensional structure of the Spanish version of the Positive and Negative Affect Schedule (PANAS) in adolescents and young adults. Psychological Assessment. 2015; 27(3):e1-e9. [DOI:10.1037/pas0000107] [PMID]

[22] Di Fabio A, Bucci O. Affective profiles in Italian high school students: Life satisfaction, psychological well-being, self-esteem, and optimism. Frontiers in Psychology. 2015; 6:1310. [DOI:10.3389/ fpsyg.2015.01310] [PMID] [PMCID]

[23] Stevanovic D, Laurent J, Lakic A. Measuring positive and negative affect and physiological hyperarousal among Serbian youth. Journal of Personality Assessment. 2013; 95(1):107-17. [DOI:10.108 0/00223891.2012.718301] [PMID]

[24] Thompson ER. Development and validation of an internationally reliable short-form of the Positive and Negative Affect Schedule (PANAS). Journal of Cross-Cultural Psychology. 2007; 38(2):227-42. [DOI:10.1177/0022022106297301]

[25] Ebesutani C, Regan J, Smith A, Reise S, Higa-McMillan C, Chorpita BF. The 10-item positive and negative affect schedule for children, child and parent shortened versions: Application of item response theory for more efficient assessment. Journal 
of Psychopathology and Behavioral Assessment. 2012; 34(2):191203. [DOI:10.1007/s10862-011-9273-2]

[26] Ioannidis CA, Siegling AB. Criterion and incremental validity of the emotion regulation questionnaire. Frontiers in Psychology. 2015; 6:247. [DOI:10.3389/fpsyg.2015.00247] [PMID] [PMCID]

[27] Lotfi M, Bahrampouri L, Amini M, Fatemitabar R, Birashk B, Entezari M, et al. [Persian adaptation of Emotion Regulation Questionnaire for Children and Adolescents (ERQ-CA) (Persian)]. Journal of Mazandaran University of Medical Sciences. 2019; 29(175):117-28.

[28] Kovacs M. The Children's Depression Inventory (CDI). Psychopharmacology Bulletin. 1985; 2:995-8.

[29] Gullone E, Taffe J. The Emotion Regulation Questionnaire for Children and Adolescents (ERQ-CA): A psychometric evaluation. Psychological Assessment. 2012; 24(2):409-17. [DOI:10.1037/ a0025777] [PMID]

[30] Dehshiri GR, Najafi M, Sheikhi M, Habibi Askarabad M. [Investigating primary psychometric properties of Children's Depression Inventory (CDI) (Pesian)]. Journal of Family Research 2009; 5(2):159-77.

[31] Spence SH, Barrett PM, Turner CM. Psychometric properties of the Spence Children's Anxiety Scale with young adolescents. Journal of Anxiety Disorders. 2003; 17(6):605-25. [DOI:10.1016/ S0887-6185(02)00236-0]

[32] Mousavi R, Moradi AR, Farzad V, Mahdavi Harsini SE, Spence S, Navabinejad Sh. Psychometric Properties of the Spence Children's Anxiety Scale with an Iranian sample. International Journal of Psychology. 2007; 1(1):17-26.

[33] Hambleton RK. The next generation of the ITC test translation and adaptation guidelines. European Journal of Psychological Assessment. 2001; 17(3):164-72. [DOI:10.1027//1015-5759.17.3.164]

[34] Epstein J, Santo RM, Guillemin F. A review of guidelines for cross-cultural adaptation of questionnaires could not bring out a consensus. Journal of Clinical Epidemiology. 2015; 68(4):435-41. [DOI:10.1016/j.jclinepi.2014.11.021] [PMID]

[35] Merz EL, Malcarne VL, Roesch SC, Ko CM, Emerson M, Roma VG, et al. Psychometric properties of Positive and Negative Affect Schedule (PANAS) original and short forms in an African American community sample. Journal of Affective Disorders. 2013; 151(3):942-9. [DOI:10.1016/j.jad.2013.08.011] [PMID] [PMCID]

[36] de Carvalho HW, Andreoli SB, Lara DR, Patrick CJ, Quintana MI, Bressan RA, et al. Structural validity and reliability of the Positive and Negative Affect Schedule (PANAS): Evidence from a large Brazilian community sample. Brazilian Journal of Psychiatry. 2013; 35(2):169-72. [DOI:10.1590/1516-4446-2012-0957] [PMID]

[37] Vera-Villarroel P, Urzua A, Jaime D, Contreras D, Zych I, CelisAtenas K, et al. Positive and Negative Affect Schedule (PANAS): Psychometric properties and discriminative capacity in several chilean samples. Evaluation \& the Health Professions. 2019; 42(4):473-97. [DOI:10.1177/0163278717745344] [PMID]

[38] Schmidt FL, Le H, Ilies R. Beyond alpha: An empirical examination of the effects of different sources of measurement error on reliability estimates for measures of individual differences constructs. Psychological Methods. 2003; 8(2):206-24. [DOI:10.1037/1082-989X.8.2.206] [PMID]
[39] Wante L, Van Beveren ML, Theuwis L, Braet C. The effects of emotion regulation strategies on positive and negative affect in early adolescents. Cognition \& Emotion. 2018; 32(5):988-1002. [D OI:10.1080/02699931.2017.1374242] [PMID]

[40] Eisner LR, Johnson SL, Carver CS. Positive affect regulation in anxiety disorders. Journal of Anxiety Disorders. 2009; 23(5):645-9. [DOI:10.1016/j.janxdis.2009.02.001] [PMID] [PMCID]

[41] Bastian VA, Burns NR, Nettelbeck T. Emotional intelligence predicts life skills, but not as well as personality and cognitive abilities. Personality and Individual Differences. 2005; 39(6):113545. [DOI:10.1016/j.paid.2005.04.006]

[42] Feldman GC, Joormann J, Johnson SL. Responses to positive affect: A self-report measure of rumination and dampening. $\mathrm{Cog}$ nitive Therapy and Research. 2008; 32(4):507-25. [DOI:10.1007] s10608-006-9083-0] [PMID] [PMCID]

[43] Siu AFY. Trait emotional intelligence and its relationships with problem behavior in Hong Kong adolescents. Personality and Individual Differences. 2009; 47(6):553-7. [DOI:10.1016/j. paid.2009.05.004]

[44] Liau AK, Liau AWL, Teoh GBS, Liau MTL. The case for emotional literacy: The influence of emotional intelligence on problem behaviours in Malaysian secondary school students. Journal of Moral Education. 2003; 32(1):51-66. [DOI:10.1080/030572402200 0073338]

[45] Patrick CJ, Bernat EM. The construct of emotion as a bridge between personality and psychopathology. In: Krueger F, Tackett JL, editors. Personality and Psychopathology. New York, NY: Guilford Press; 2006.

[46] Clark LA. Temperament as a unifying basis for personality and psychopathology. Journal of Abnormal Psychology. 2005; 114(4):505-21. [DOI:10.1037/0021-843X.114.4.505] [PMID] 